OPEN ACCESS

Edited by:

Uffe Kock Wiil,

University of Southern

Denmark, Denmark

Reviewed by:

Mathijs Lucassen,

The Open University, United Kingdom

Nele A. J. De Witte,

Thomas More University of Applied

Sciences, Belgium

*Correspondence:

lan Chard

i.chard18@imperial.ac.uk

Specialty section: This article was submitted to

Digital Mental Health,

a section of the journal

Frontiers in Digital Health

Received: 23 December 2021

Accepted: 28 January 2022

Published: 25 February 2022

Citation:

Chard I and van Zalk N (2022) Virtual Reality Exposure Therapy for Treating

Social Anxiety: A Scoping Review of

Treatment Designs and Adaptation to

Stuttering.

Front. Digit. Health 4:842460.

doi: 10.3389/fdgth.2022.842460

\section{Virtual Reality Exposure Therapy for Treating Social Anxiety: A Scoping Review of Treatment Designs and Adaptation to Stuttering}

\author{
lan Chard ${ }^{*}$ and Nejra van Zalk \\ Design Psychology Lab, Dyson School of Design Engineering, Imperial College London, London, United Kingdom
}

Virtual Reality Exposure Therapy (VRET) has been shown to be an effective technique for reducing social anxiety. People who stutter are at greater risk of developing heightened social anxiety. Cognitive behavior therapy protocols have shown promise in reducing social anxiety in people who stutter, but no studies have investigated VRET targeting social anxiety associated with stuttering. The aim of the current review is to provide an overview of VRET techniques used to treat social anxiety and insights into how these techniques might be adopted in the case of comorbid stuttering and social anxiety. Twelve studies were reviewed to understand key distinctions in VRET protocols used to treat social anxiety. Distinctions include exercises targeting public speaking vs. general social anxiety, computer-generated virtual environments vs. $360^{\circ}$ video, and therapist guided vs. automated VRET. Based on the review findings, we propose how certain features could be applied in the case of stuttering. Virtual therapists, inhibitory learning techniques and integration into speech therapy may be suitable ways to tailor VRET. Regardless of these different techniques, VRET should consider the situations and cognitive-behavioral processes that underlie the experience of social anxiety amongst people who stutter.

Keywords: social anxiety, social phobia, stuttering, stammering, virtual reality, VRET

\section{INTRODUCTION}

Stuttering is a developmental speech disorder characterized by the involuntary disruption to the fluent production of speech (1). Approximately $5 \%$ of people will experience stuttering in their lifetime, with roughly $1 \%$ prevalence within the overall population at any one time (2-4). Whilst many children who stutter recover naturally in early childhood, a significant proportion continue to stutter chronically (5).

A large body of evidence links stuttering to heightened levels of social anxiety $(6,7)$. Social anxiety is characterized as "a marked, or intense, fear or anxiety of social situations in which the individual may be scrutinized by others" [(1), p. 202]. For some people who stutter (PWS), this will manifest as subclinical social anxiety (or shyness) which is related but considered distinct from a clinical diagnosis of social anxiety disorder (SAD) (8). However, PWS are also at greater risk of developing SAD compared to fluent speakers. Approximately $46 \%$ of PWS are estimated to meet diagnostic criteria for SAD as opposed to $4 \%$ of fluent speakers (9). Core to the experience of social anxiety is the expectation of negative evaluation from others, and the overestimation 
of the consequences this will have (10). For PWS, social anxiety is embedded in speech and communication and revolves around the expectation that others will react negatively to one's stutter, and the overestimation of the consequences this will have $(10,11)$. This can result in greater speech dissatisfaction and avoidance of speaking situations $(12,13)$.

Social anxiety arises irrespective of speech fluency levels (12), therefore it is unsurprising that speech therapy techniques have limited effects on reducing social anxiety (14). It is therefore imperative that effective treatments be made available to PWS targeting social anxiety. PWS are likely to benefit from existing treatment protocols given their experience is rooted in the same principles of social anxiety such as fear of negative evaluation. However, tailoring treatment to consider the stuttering-specific nature of social anxiety will ensure treatment is as relevant as possible. As such, treatments should consider the cognitive processes, thoughts, and behaviors associated with social anxiety in stuttering (11), as well as situational factors and practical considerations. Cognitive Behavioral Therapy (CBT) is considered the gold-standard treatment for anxiety disorders (15) and several studies have investigated its use for PWS (1618 ), with some adopting protocols which consider stutteringspecific processes $(14,19-24)$. This includes targeting speechrelated fears, and the safety behaviors and expectations that are common amongst PWS. To the best of our knowledge, only three randomized trials have been conducted to date. In one study, CBT eliminated all SAD diagnoses in the sample and was associated with a decrease in social anxiety (14). Another study adopted a fully automated, online version of CBT, finding it was equally as effective as in vivo $\mathrm{CBT}$ at reducing social anxiety levels (23). The third trial also used automated CBT, demonstrating that when integrated into speech restructuring, it can aid the long-term maintenance of speech outcomes (22). Tailored CBT is therefore one appropriate method for targeting social anxiety associated with stuttering and may support retention of outcomes from speech therapy.

A central component of CBT is exposure therapy, a behavioral technique that involves immersing the patient in a scenario they fear, with the aim of adapting memories associated with feared stimuli using corrective information. Experiencing feared situations is considered key to the activation of mental representations in order to challenge unhelpful thoughts and promote new learning (25). This is usually done by confronting real feared stimuli (in vivo) or by visualizing them (imaginal). As the feared situation offers a strong forum for performing the cognitive elements of treatment, exposure is often considered as a standalone treatment (25), and some evidence suggests it is no less inferior than CBT (26).

Two separate theoretical models lay out alternative arguments regarding how exposure reduces anxiety and informs different treatment techniques. According to the Emotional Processing Theory (27), when corrective information is presented alongside the feared stimulus, it can overwrite the existing fear structure

\footnotetext{
Abbreviations: VRET, virtual reality exposure therapy; PWS, people who stutter; $\mathrm{SAD}$, social anxiety disorder; $\mathrm{CBT}$, cognitive behavioral therapy; VR, virtual reality; HMD, head-mounted display; ACT, acceptance and commitment therapy.
}

within memory. Within- and between-session habituation are the primary sources of this information. Because the reduction of anxiety runs counter to the expectations an individual has about their feared stimulus, they begin to associate the stimulus with a lower response. Thus, habituation is considered the cornerstone to successful anxiety reduction, determining progress through exercises. The Inhibitory Learning Model (28) suggests that a broader learning approach might explain how exposure operates. It purports that when patients are exposed to their feared stimulus along with corrective information, they learn how to inhibit the existing fear structure through learning new associations, rather than overwriting it. The primary technique associated with this theory is expectancy violation, in which exposure exercises are designed to violate the beliefs that patients may have about expected outcomes and promote the learning of new expectations. Both theoretical approaches have been widely used in exposure studies.

Exposure therapy may also be particularly relevant for stuttering. For example, the inhibitory learning model outlines strategies to deal with continued negative reactions that can occur post-treatment, and the often resulting reacquisition of anxiety (29). Exposure also provides the ideal medium to phase out stuttering-specific safety behaviors such as decreased verbal participation, avoidance of troublesome words and rehearsal of utterances before speaking (13). Exposure scenarios can be designed around these behaviors, so that PWS are forced to confront their feared situations which is necessary to learn new associations (29). Exposure is commonly used already in many speech and language programs. However, the general aim is different as it is used to practice new speech techniques in increasingly difficult situations.

In recent years, virtual reality (VR) has emerged as a promising tool to conduct exposure therapy. Virtual Reality Exposure Therapy (VRET) uses virtual environments to expose patients to anxiety-inducing stimuli. Typically, these stimuli are presented using a head-mounted display (HMD), which uses motion tracking and binocular graphics with a wide field of view to provide an immersive experience.

The ability of VR to create on-demand experiences has made it an appealing medium for exposure therapy, which requires repeated experience of fear-inducing situations. In the case of social anxiety, VRET involves the confrontation of social stimuli that provoke fear of public scrutiny and negative evaluation from others. Scenarios might include performative elements such as public speaking, or interactive elements such as speaking to those with authority and ordering food or drink. Recreating believable social interactions in a virtual environment is one of the greatest challenges for VRET, yet virtual social environments have proved to be effective in replicating human reactions to real social environments. Numerous studies have demonstrated heightened self-reported social anxiety and physiological responses when exposed to social environments in VR (30-34). Research has also showed that typical safety behaviors are observable in virtual environments $(35,36)$.

To date, there have been no robust assessments of exposure therapy for reducing social anxiety amongst PWS. Two studies begin to elucidate exposure's efficacy amongst PWS, one using an 
in vivo protocol (37) and the other using VRET (38). Participants in the in vivo study received 10 exposure sessions targeting public speaking fears, following the emotional processing approach. Exercises were also adapted to stuttering by including words designed to induce anxiety into the speeches. In the VRET study, participants received two exposure sessions targeting public speaking fears, but it is unclear whether this followed either the emotional processing or inhibitory learning approach to exposure. Additionally, participants were able to retreat to a "chill session" if they became too anxious which may have the inadvertent effect of teaching avoidant behaviors. Both studies claimed successful outcomes from exposure. Whilst the in vivo study did observe a reduction in social anxiety symptoms from pre- to post-treatment, this was not statistically analyzed. In addition, the VRET study lacked validated measures for social anxiety symptoms and did not provide outcome data. Despite the promising findings, methodological issues preclude conclusions regarding the effectiveness of stuttering-specific exposure therapy.

However, there is more robust evidence backing the use of VRET for social anxiety in the wider population. Three meta-analyses have examined the effects of VRET on social anxiety (39-41). Several others have examined the use of VRET more generally in treating various anxiety disorders, including SAD (42-45). Whilst these meta-analyses provide a helpful indicator of VRET's efficacy, they do not expand on the different approaches adopted in treatment protocols, and the key distinctions of treatment and study designs. One of the above meta-analyses covers some of these distinctions in their analyses (41). Findings showed that both standalone VRET and VRET integrated into psychotherapy were associated with improved treatment outcome. Similarly, VRET was effective for both participants with a SAD diagnosis, or a diagnosis of public speaking anxiety. However, many questions remain regarding the implementation of VRET and the processes that potentially make it effective. Analyses also covered the influence of several factors including number of VR sessions, total number of sessions, and ratio of VR sessions to total sessions, demonstrating no influence of these factors. Due to increasingly sophisticated technology, VRET can take many shapes and forms. This includes using smartphone-based VR $(46,47), 360^{\circ}$ video for virtual environments (46-48), and virtual therapists (48). Yet no review paper comprehensively covers the key differences in treatment design. Understanding the comparative value of different techniques adopted in VRET is thus necessary to keep improving treatment design.

Given the suitability of exposure for PWS, the growth of VRET research, and the research gap concerning stuttering, it is important to consider how best to adapt treatment techniques for this population. The aim of the current study is therefore to provide further insights into how VRET might be adopted in the case of comorbid stuttering and social anxiety. Nevertheless, there is limited systematic research on the various techniques and approaches adopted in VRET protocols targeting social anxiety. One objective is therefore to provide a comprehensive review of the different approaches and techniques used in VRET design for adults. Although it would be useful to investigate VRET for non-adult populations, the child and adolescent literature is relatively limited, and developmental trajectories of social anxiety throughout childhood and adolescence preclude using the same CBT treatment protocols for adults and children who stutter [see e.g., (19) and (24) for adolescent and adult protocols, respectively], making comparisons difficult. Findings from this scoping review will outline distinctions in treatment designs adopted in adult VRET trials, and key findings from these studies. These approaches will be discussed in the context of stuttering. Whilst no rigorous trials have explored VRET for reducing social anxiety associated with stuttering, this paper will build on the knowledge gained from previous work and discuss what techniques are likely to be particularly suited to PWS.

\section{METHODS}

\section{Scoping Review}

Given the acceleration of VRET research targeting social anxiety, a scoping review was chosen for the current paper to provide the information needed to conduct a qualitative synthesis of relevant literature, and to discuss the comparative value of different VRET techniques including considerations for adopting these for stuttering. The Preferred Reporting Items for Systematic reviews and Meta-Analyses extension for Scoping Review (PRISMA-ScR) guidelines were followed (49). No review protocol was created for this review.

\section{Search Strategy}

Systematic literature searches were performed on September 22, 2021, using three databases: Web of Science, Scopus, and PsycINFO/PsycARTICLES. Search terms were primarily aimed at finding VRET trials targeting social anxiety, but also any possible developments in exposure related to stuttering. For each database, three searches were conducted. First, [(Virtual Reality OR VR) AND (Social anxiety OR social phobia OR social anxiety disorder OR SAD) AND (Therapy OR Treatment)]. Second, [(Virtual Reality OR VR) AND (Stutter* OR Stammer*) AND (Therapy OR Treatment)]. Third, [Exposure AND (Therapy OR Treatment) AND (Stutter* OR Stammer*)]. Supplementary material for complete search strategy using PsycINFO/PsycARTICLES is provided in Appendix A and was adapted for all database searches. Relevant studies identified in previous literature searches unrelated to the current scoping review were also included.

\section{Eligibility}

Studies were included if they met the following eligibility criteria: (1) study used VRET aimed at reducing social anxiety symptoms, (2) study design included at least one comparison condition such as in vivo exposure/waitlist control, in addition to the experimental VRET condition, (3) comparison condition did not include VRET, (4) VRET was not combined with any medication, (5) study participants were adults, (6) study used random/quasirandom assignment/participant matching, (7) study used at least one validated and reliable measure of social anxiety symptoms, (8) report included sufficient statistical analysis-means/SD for each group, (9) study was published in a peer-reviewed journal 


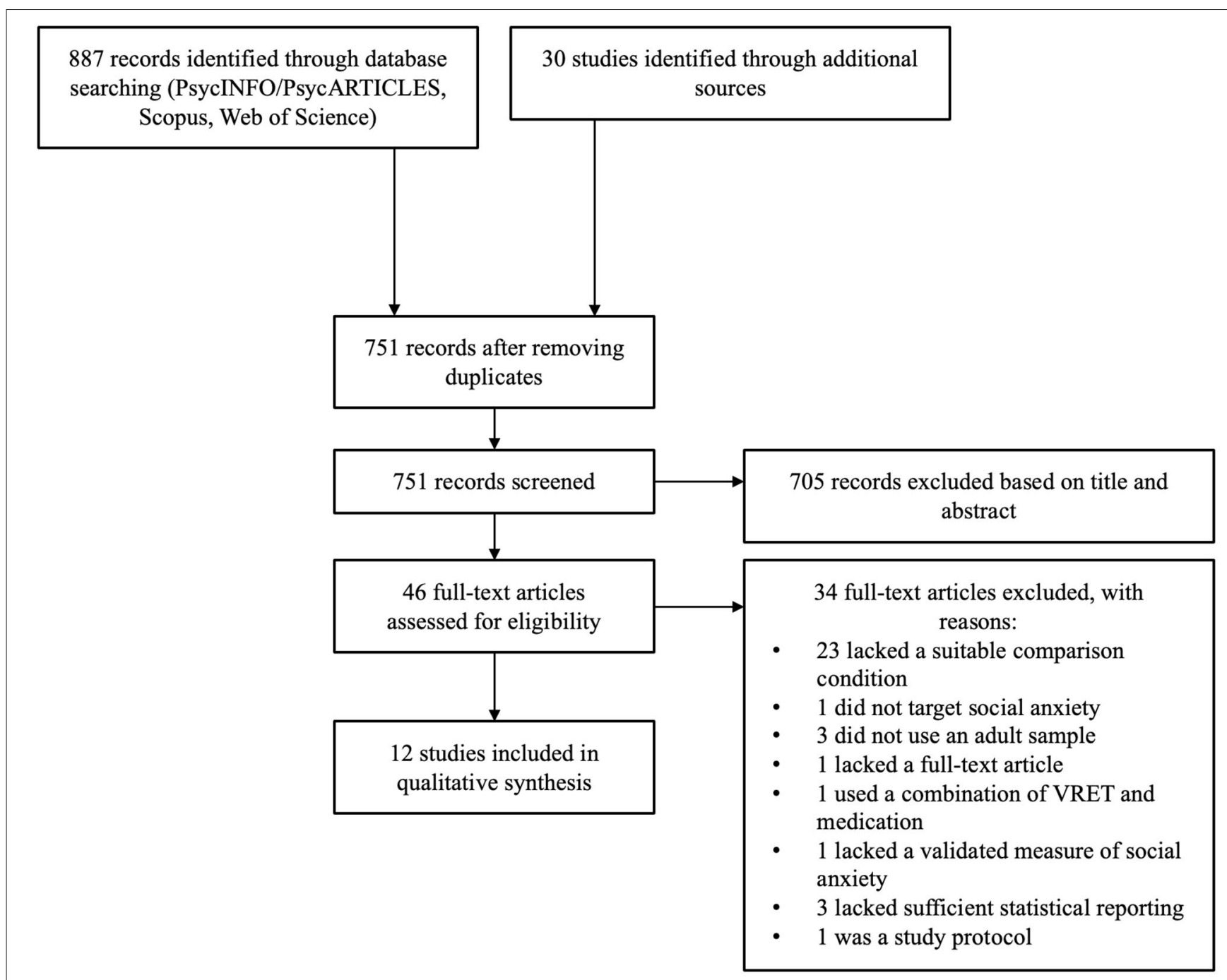

FIGURE 1 | PRISMA-ScR diagram.

article (no book chapters or dissertations), (10) study was published in English, and (11) full-text was available. Date of publication was not considered in the eligibility criteria so that all VRET protocols in the context of social anxiety were considered.

\section{Study Selection}

The first author was responsible for screening each record retrieved. Literature search results were initially exported into EndNote, and duplicates were deleted. Primary screening included reviewing the titles and abstracts of all results. Those which were irrelevant or did not fit eligibility criteria were excluded at this stage. Studies were then reviewed as part of secondary screening for thorough assessment against eligibility criteria. The reason for excluding each study not meeting criteria was noted. A full breakdown of this process is outlined in the PRISMA flowchart (Figure 1).

\section{Data Extraction}

The data were obtained and summarized by the first author. A data extraction table was generated to note key features of VRET protocols for each eligible study. The following data were extracted: (1) authors and publication date, (2) sample size and no. of participants in each condition, (3) type of participant assignment, (4) mean age, (5) whether participants reported clinical/subclinical level of social anxiety, (6) social anxiety measures, (7) type of treatment/control used in comparison condition(s), (8) target of VRET (social anxiety or public speaking anxiety), (9) whether VRET was standalone integrated into CBT, (10) whether VRET followed emotional processing or inhibitory learning approach to exposure, (11) number of treatment sessions, (12) time from pre- to post-treatment (and follow-up if included), (13) whether VRET was delivered remotely or in-person, (14) whether there was a real or virtual therapist, (15) whether virtual environments were computergenerated or $360^{\circ}$ video, (16) whether VRET was delivered using 
a HMD or smartphone HMD, and (17) whether analyses were based on completers or intention to treat. For each eligible paper, details of the treatment protocol, and key findings were also noted.

\section{RESULTS: KEY DISTINCTIONS IN VRET STUDIES TARGETING SOCIAL ANXIETY}

A total of 12 studies were eligible for this scoping review following the screening process. Table 1 provides a summary of the findings from this review. Studies were conducted in the USA (4), Canada (2), Israel (2), The Netherlands (1), France (1), Sweden (1) and the UK (1). Studies were published over a 19-year period between 2002 and 2021, with the quality of VR equipment varying across the papers. The mean age of participants ranged between 23 and 42 years but was skewed toward younger adults. Key distinctions in treatment protocols that have been adopted in these studies are outlined in more detail below and in Table 2 .

\section{Clinical vs. Subclinical Social Anxiety}

All the studies in this analysis target adults with elevated social anxiety levels and discuss the relevance of their findings within a clinical context. However, whilst some include a clinical diagnosis of SAD or public speaking anxiety in their eligibility criteria $(47,48,50-52,54-56)$, some only use elevated social anxiety $(46,53,57,58)$. The investigation of non-clinical samples is likely to become more important with the rise of self-guided, remote treatments that are not designed as clinical interventions, but as accessible treatments for subclinical use.

Both VRET protocols targeting clinical and subclinical levels of social anxiety are likely to have suitable applications for stuttering. Clinical VRET could be useful for the significant subset of PWS who have a diagnosis of SAD (9), whilst generally elevated levels of social anxiety $(6,7)$ may make subclinical VRET an appealing option for some. However, subclinical VRET may be particularly suited for delivery alongside speech therapy. Some authors have suggested that all PWS receiving speech therapy should also receive psychological treatment (23), given findings that mental health conditions can disrupt the progress made from speech therapy (72). Such is the highly entwined nature of comorbid stuttering and social anxiety, that cognitive and behavioral change are considered necessary for managing stuttering (73). Clinical VRET is unlikely to be appropriate in this context but a less substantial form of VRET targeting subclinical social anxiety may be more suited. Integrating subclinical VRET into speech therapy may also be one strategy to increase the uptake of social anxiety treatment amongst PWS, given this is often the first port of call. VRET is also far simpler and cheaper to administer than in vivo exposure, which could reduce the burden of delivering additional treatment.

\section{Public Speaking vs. General Social Anxiety}

Public speaking fears were targeted in 7 of the 12 studies (46, $47,50,51,53,57,58)$. Given that a significant proportion of people only experience performance-only social anxiety (74), it is unsurprising that most studies target these scenarios only. Performance scenarios are easier to develop and conduct as they do not include interactions and therefore remove the need for turn-taking conversation that can be difficult to recreate realistically in VRET.

The earliest of these studies (53) used an auditorium scenario and made tasks increasingly difficult as participants progressed. This involved increasingly larger audiences and audience members talking and asking the participant to speak louder. VRET was associated with a decrease in social anxiety scores, but this was not true for all measures. At post-treatment, there was little difference between the VRET and waitlist conditions. A more recent study also manipulated the setting for the public speaking task (47). Using an auditorium, wedding reception and meeting room, they changed the size of the audience and the difficulty of the task. Findings were more encouraging, showing a significantly greater decrease on all social anxiety measures compared to the waitlist condition. Two studies adapted public speaking tasks to the individual, changing the scenario, audience reactions, and task details in accordance with each participant's fear hierarchy $(50,58)$. Both studies showed comparable reductions on most social anxiety measures compared to in vivo exposure therapy. However, they also showed that VRET did not reduce fear of negative evaluation to the same extent as in vivo exposure therapy. Follow-up assessments of participants in these studies showed treatment gains were maintained long-term $(51,57)$. Another recent study further explored how the nature of the public speaking task might influence treatment efficacy by comparing VRET protocols using audiences or empty rooms (46). In both conditions, the room size increased throughout sessions, and the audience size also grew in the audience condition. Both treatments were effective across all measures; however, the empty room version failed to show superiority over waitlist with regards to fear of negative evaluation. Together, these findings support the efficacy of VRET targeting public speaking fears, but some protocols are limited in reducing fear of negative evaluation, a core element of social anxiety. Inclusion of social cues is likely necessary for this (46), however cannot explain the null findings from other studies $(50,58)$.

Whilst individuals experiencing social anxiety more generally are likely to experience public speaking anxiety and may benefit somewhat from the above protocols, a more varied treatment protocol targeting a variety of scenarios is more appropriate. According to the Diagnostic and Statistical Manual of Mental Disorders (DSM-5), the "Performance only" subtype is considered distinct from general SAD (1). Targeting feared stimuli in multiple contexts is also considered key for maximizing exposure according to the inhibitory learning theory (29). The other five studies that were reviewed targeted a variety of social evaluative scenarios $(48,52,54-56)$.

All studies used environments targeting different facets of social anxiety such as performance, intimacy, assertiveness, scrutiny, and observation anxiety. Three of these studies adopted the same set of stimuli $(52,55,56)$. Exposure exercises replicated a public speaking scenario, dinner party, busy café and shoe store targeting performance, intimacy, scrutiny, and assertiveness anxiety respectively. Treatment gains from these protocols were shown to be superior to waitlist condition $(52,56)$ and 
TABLE 1 | Studies included in scoping review, methodological details, and key features of VRET protocols.

\begin{tabular}{|c|c|c|c|c|c|c|c|c|c|c|c|c|c|c|}
\hline References & $\begin{array}{l}\text { Participant } \\
\text { age }(M)\end{array}$ & $\begin{array}{l}\text { VRET } \\
\text { (N) }\end{array}$ & $\begin{array}{l}\text { Active } \\
\text { control }\end{array}$ & $\begin{array}{l}\text { Waitlist } \\
\text { control }\end{array}$ & $\begin{array}{l}\text { Comparison } \\
\text { condition }\end{array}$ & $\begin{array}{l}\text { Main } \\
\text { variables of } \\
\text { interest }\end{array}$ & $\begin{array}{l}\text { Clinical } \\
\text { diagnosis } \\
\text { required }\end{array}$ & $\begin{array}{l}\text { Target of } \\
\text { VRET }\end{array}$ & $\begin{array}{l}\text { Exposure } \\
\text { technique }\end{array}$ & $\begin{array}{l}\text { Type of virtual } \\
\text { environment }\end{array}$ & $\begin{array}{l}\text { Facial } \\
\text { expressions } \\
\text { in } \\
\text { audience }\end{array}$ & HMD type & $\begin{array}{l}\text { Therapist } \\
\text { type }\end{array}$ & $\begin{array}{l}\text { Integration } \\
\text { into CBT }\end{array}$ \\
\hline $\begin{array}{l}\text { Anderson et al. } \\
\text { (50) }\end{array}$ & 39 & 30 & 39 & 28 & IVET, WL & $\begin{array}{l}\text { FNE-B, PRCS, peak } \\
\text { anxiety during } \\
\text { speech, speech } \\
\text { length }\end{array}$ & Yes & PSA & Unknown & $\begin{array}{l}\text { Computer- } \\
\text { generated }\end{array}$ & Yes & Unknown & Real & Yes \\
\hline $\begin{array}{l}\text { Anderson et al. } \\
\text { (51) }\end{array}$ & 42 & 13 & 15 & & IVET & $\begin{array}{l}\text { FNE-B, PRCS, peak } \\
\text { anxiety during } \\
\text { speech, speech } \\
\text { length }\end{array}$ & Yes & PSA & Unknown & $\begin{array}{l}\text { Computer- } \\
\text { generated }\end{array}$ & Yes & Unknown & Real & Yes \\
\hline $\begin{array}{l}\text { Bouchard et al. } \\
\text { (52) }\end{array}$ & 34.5 & 17 & 22 & 20 & CBT-IV, WL & $\begin{array}{l}\text { LSAS-SR, SPS, } \\
\text { SIAS, FNE, BAT } \\
\text { (SPRS) }\end{array}$ & Yes & General SA & $\begin{array}{l}\text { Inhibitory } \\
\text { learning }\end{array}$ & $\begin{array}{l}\text { Computer- } \\
\text { generated }\end{array}$ & Yes & $\begin{array}{l}\text { Research- } \\
\text { grade } \\
\text { HMD }\end{array}$ & Real & Yes \\
\hline Harris et al. (53) & Unknown & 8 & & 6 & WL & $\begin{array}{l}\text { LSAS, PRCS, STAI, } \\
\text { ATPS, HR during } \\
\text { voice sample }\end{array}$ & No & PSA & Unknown & Unknown & Unknown & $\begin{array}{l}\text { Research- } \\
\text { grade } \\
\text { HMD }\end{array}$ & Real & No \\
\hline $\begin{array}{l}\text { Kampmann et al. } \\
(39,54)\end{array}$ & 36.9 & 20 & 20 & 20 & IVET, WL & $\begin{array}{l}\text { LSAS-SR, FNE-B, } \\
\text { speech duration, } \\
\text { speech performance }\end{array}$ & Yes & General SA & $\begin{array}{l}\text { Emotional } \\
\text { processing }\end{array}$ & $\begin{array}{l}\text { Computer- } \\
\text { generated }\end{array}$ & Unknown & $\begin{array}{l}\text { Research- } \\
\text { grade } \\
\text { HMD }\end{array}$ & Real & No \\
\hline Klinger et al. (55) & 32 & 18 & 18 & & CBT-IV & LSAS, RAS & Yes & General SA & Unknown & $\begin{array}{l}\text { Computer- } \\
\text { generated }\end{array}$ & Yes & Monitor & Real & Unknown \\
\hline Lindner et al. (47) & 31.4 & 25 & & 25 & WL & $\begin{array}{l}\text { PSAS, LSAS-SR, } \\
\text { FNE-B }\end{array}$ & Yes & PSA & $\begin{array}{l}\text { Inhibitory } \\
\text { learning }\end{array}$ & $360^{\circ}$ video & Unknown & $\begin{array}{l}\text { Smartphone } \\
\text { HMD }\end{array}$ & Real & No \\
\hline Reeves et al. (46) & 26.1 & $\begin{array}{c}17 \\
\text { (Audience), } \\
16 \\
\text { (Empty } \\
\text { room) }\end{array}$ & & 18 & WL & $\begin{array}{l}\text { PSAS, LSAS-SR, } \\
\text { FNE-B, speech } \\
\text { duration }\end{array}$ & No & PSA & Unknown & $360^{\circ}$ video & Yes & $\begin{array}{l}\text { Smartphone } \\
\text { HMD }\end{array}$ & None & No \\
\hline $\begin{array}{l}\text { Robillard et al. } \\
\text { (56) }\end{array}$ & 34.9 & 14 & 16 & 15 & CBT-IV, WL & $\begin{array}{l}\text { LSAS, SPS, ASC-P, } \\
\text { ASC-C, FNE }\end{array}$ & Yes & General SA & Unknown & $\begin{array}{l}\text { Computer- } \\
\text { generated }\end{array}$ & Yes & $\begin{array}{l}\text { Research- } \\
\text { grade } \\
\text { HMD }\end{array}$ & Real & Yes \\
\hline Safir et al. (57) & 27 & 25 & 24 & & CBT-IV & $\begin{array}{l}\text { LSAS, SSPS, FNE, } \\
\text { self- and } \\
\text { observer-rated } \\
\text { anxiety during } \\
\text { speech }\end{array}$ & No & PSA & Unknown & $\begin{array}{l}\text { Computer- } \\
\text { generated }\end{array}$ & Unknown & $\begin{array}{l}\text { Research- } \\
\text { grade } \\
\text { HMD }\end{array}$ & Real & Yes \\
\hline Wallach et al. (58) & 27 & 28 & 30 & 30 & CBT-IV, WL & $\begin{array}{l}\text { LSAS, SSPS, FNE, } \\
\text { self- and } \\
\text { observer-rated } \\
\text { anxiety during } \\
\text { speech }\end{array}$ & No & PSA & Unknown & $\begin{array}{l}\text { Computer- } \\
\text { generated }\end{array}$ & Unknown & $\begin{array}{l}\text { Research- } \\
\text { grade } \\
\text { HMD }\end{array}$ & Real & Yes \\
\hline Zainal et al. (48) & 23.3 & 26 & & 18 & WL & SPDQ, SIAS, MASI & Yes & General SA & $\begin{array}{l}\text { Emotional } \\
\text { processing }\end{array}$ & $360^{\circ}$ video & Unknown & $\begin{array}{l}\text { Consumer } \\
\text { HMD }\end{array}$ & Virtual & No \\
\hline
\end{tabular}

IVET, in vivo exposure therapy; WL, waitlist; CBT-IV, CBT with in vivo exposure therapy; FNE-B, fear of negative evaluation-brief form (59); PRCS, personal report of confidence as a speaker (60); LSAS-SR, Liebowitz social anxiety scale-self reported version (61): SPS, social phobia scale (62): SIAS, social interaction anxiety scale (62); FNE, fear of negative evaluation (63); BAT, behavioral assessment task; SPRS, social performance rating scale (64); LSAS, Liebowitz social anxiety scale (65); RAS, Rathus assertiveness schedule (66); PSAS, public speaking anxiety scale (67): ASC-P, appraisal of social concerns - probability subscale (68); ASC-C, appraisal of social concerns - consequences subscale (68); SSPS, self-statements during public speaking (69); SPDQ, social phobia diagnostic questionnaire (70); MASI, measure of anxiety in selection interviews (71); PSA, public speaking anxiety; SA, social anxiety. 
TABLE 2 | Key features of studies included in the scoping review.

\begin{tabular}{ll}
\hline Study & Key features \\
\hline Anderson et al. (50, 51) & - Participants had a clinical diagnosis of SAD \\
& - Exercises targeted public speaking anxiety using a virtual conference room ( $\sim 5$ audience members), a virtual classroom ( 35 audience \\
& members), and a virtual auditorium (100+ audience members). Scenario, audience reactions, and task details were adapted in accordance \\
& with participant's fear hierarchy \\
- Exposure techniques not specified & - Computer-generated virtual environments \\
- Virtual avatars displayed facial expressions & - HMD type not specified \\
- & Assisted by therapist-2 clinical psychologists, 3 doctoral students \\
- & VRET integrated into CBT
\end{tabular}

Bouchard et al. (52)

- Participants had a clinical diagnosis of SAD

- Exercises targeted general social anxiety using a public speaking scenario, dinner party, busy café and shoe store targeting performance, intimacy, scrutiny, and assertiveness anxiety respectively

- Inhibitory learning approach

- Computer-generated virtual environments-low-fidelity

- Virtual avatars displayed facial expressions

- Research-grade HMD-eMagin z800

- Assisted by therapist-doctoral students with 1-year CBT experience

- VRET integrated into CBT

Harris et al. (53) - Participants did not have a clinical diagnosis of SAD, but had elevated levels of social anxiety

- Exercises targeted public speaking anxiety using an auditorium. Exercises were made increasingly difficult through larger audiences and audience members talking and asking the participant to speak louder

- Exposure techniques not specified

- Type of virtual environment not specified

- Use of facial expressions not specified

- Research-grade HMD-Virtual-I/0

- Assisted by therapist-clinical psychologist

- VRET delivered as a standalone treatment

Kampmann et al. (54) - Participants had a clinical diagnosis of SAD

- Exercises targeted general social anxiety and placed an emphasis on verbal interaction. Exercises were based on either one-to-one or group scenarios and included giving a talk in front of an audience followed by questions from the audience, talking to a stranger, buying and returning clothes, attending a job interview, being interviewed by journalists, dining in a restaurant with a friend, and having a blind date

- Emotional processing approach-participants rated their anxiety level before, during and after each exercise, and repeated it another time if anxiety did not decrease

- Computer-generated virtual environments-high-fidelity

- Use of facial expressions not specified

- Research-grade HMD-nVisor SX

- Assisted by therapist-clinical psychologists, clinical psychology master's degree students

- VRET delivered as a standalone treatment

Klinger et al. (55) - Participants had a clinical diagnosis of SAD

- Exercises targeted general social anxiety using a public speaking scenario, dinner party, busy café and shoe store targeting performance, intimacy, scrutiny, and assertiveness anxiety respectively

- Exposure techniques not specified

- Computer-generated virtual environments-low-fidelity

- Virtual avatars displayed facial expressions

- Exposure conducted using a computer monitor

- Assisted by therapist-clinical psychologist

- Unknown whether VRET was integrated into CBT

Lindner et al. (47) - Participants had clinical levels of public speaking anxiety

- Exercises targeted public speaking anxiety using an auditorium, wedding reception and meeting room. Audience size and task difficulty was modified throughout exercises.

- Inhibitory learning approach

- $360^{\circ}$ video virtual environments

- Use of facial expressions not specified

- Smartphone-based HMD-Samsung Gear VR

- Assisted by therapist--1 clinical psychologist, 3 clinical psychology master's degree students

- VRET delivered as a standalone treatment 
TABLE 2 | Continued

\begin{tabular}{|c|c|}
\hline Study & Key features \\
\hline Reeves et al. (46) & $\begin{array}{l}\text { - Participants had subclinical levels of social anxiety } \\
\text { - Exercises targeted public speaking anxiety, but participants spoke either in front of an audience or an empty room. In both conditions, the } \\
\text { room size increased throughout exercises. In the audience condition, audience size also increased. } \\
\text { - Exposure techniques not specified } \\
\text { - } 360^{\circ} \text { video virtual environments } \\
\text { - Virtual avatars displayed facial expressions } \\
\text { - Smartphone-based HMD-Samsung Gear VR } \\
\text { - No therapist } \\
\text { - VRET delivered as a standalone treatment }\end{array}$ \\
\hline Robillard et al. (56) & $\begin{array}{l}\text { - Participants had subclinical levels of public speaking anxiety } \\
\text { - Exercises targeted general social anxiety using a public speaking scenario, dinner party, busy café and shoe store targeting performance, } \\
\text { - Exposure techniques not specified } \\
\text { - Computer-generated virtual environments-low-fidelity } \\
\text { - Virtual avatars displayed facial expressions } \\
\text { - Research-grade HMD-eMagin z800 } \\
\text { - Assisted by therapist-training not specified } \\
\text { - VRET integrated into CBT }\end{array}$ \\
\hline $\begin{array}{l}\text { Safir et al. (57) and } \\
\text { Wallach et al. (58) }\end{array}$ & $\begin{array}{l}\text { - Participants had subclinical levels of public speaking anxiety } \\
\text { - Exercises targeted public speaking anxiety using a large audience. Audience reactions and task details were adapted in accordance with } \\
\text { - Exposure techniques not specified } \\
\text { - Computer-generated virtual environments } \\
\text { - Use of facial expressions not specified } \\
\text { - Research-grade HMD-VFX3D } \\
\text { - Assisted by therapist-clinical psychology students } \\
\text { - VRET integrated into CBT }\end{array}$ \\
\hline Zainal et al. (48) & $\begin{array}{l}\text { - Participants had a clinical diagnosis of SAD } \\
\text { - Exercises targeted general social anxiety using a formal job interview and informal dinner party. Performance, intimacy, assertiveness, and } \\
\text { observation anxiety were targeted. } \\
\text { - Emotional processing approach-participants moved onto the next exposure exercise if anxiety had decreased by } 50 \% \text { across three } \\
\text { consecutive attempts or whether ratings before an attempt were sufficiently low } \\
\text { - } 360^{\circ} \text { video virtual environments } \\
\text { - Use of facial expressions not specified } \\
\text { - Consumer HMD-Pico Goblin } \\
\text { - Assisted by virtual therapist-used voiceover to outline principles of exposure, provide instructions and to coach participants through exercises } \\
\text { - VRET delivered as a standalone treatment }\end{array}$ \\
\hline
\end{tabular}

comparable to in vivo exposure therapy $(52,55)$. One of the studies also found superiority of VRET over in vivo exposure at both post-treatment and 6-month follow-up on Liebowitz Social Anxiety Scale and Social Phobia Scale scores (52). Another study only used two scenarios (formal job interview and informal dinner party) but the content changed between exercises to target different domains of social anxiety including performance, intimacy, assertiveness, and observation anxiety (48). Again, treatment gains from VRET were found to be superior to the waitlist condition on all measures. The last of these studies placed an emphasis on verbal interaction through their scenarios, noting previous protocols had been limited (54). This is important for VRET targeting social anxiety more generally, given that verbal interaction forms a central part of social fears. Participants were exposed to a variety of environments that were based on either one-to-one or group scenarios. Whilst VRET decreased social anxiety significantly compared to waitlist, and was comparable with in vivo exposure, VRET failed to reduce fear of negative evaluation. Given the mixed findings regarding fear of negative evaluation, it is unlikely that the domains of social anxiety that VRET targets influence the reduction of this construct.

VRET targeting general social fears will be more appropriate for the majority of PWS. Findings from a recent preprint suggest that, besides speaking on the phone, situational factors influencing social anxiety were largely similar in PWS and fluent speakers, including those with SAD (75). Findings from another preprint also showed that general speech-related fears are common across PWS (76). VRET targeting a variety of social anxiety domains is therefore likely to have a more efficacious outcome than performance only VRET. This is supported by findings suggesting $\sim 22-46 \%$ of PWS meet diagnostic criteria for $\operatorname{SAD}(9,77)$. However, there are some situational factors that should be considered when designing VRET protocols. Findings from both of the above preprints suggest that fear of speaking 
on the telephone is particularly elevated amongst PWS and may constitute a distinguishable sub-type of social anxiety amongst PWS $(75,76)$. A telephone-only treatment may therefore be appropriate for some PWS. However, as the nature of social anxiety has been shown to vary across PWS, one challenge will be to adapt VRET to individual needs.

Other than situational factors, stuttering-specific cognitivebehavioral processes should also be considered in VRET design. Thoughts and expectations are likely to revolve around speech and the perceived costs that stuttering will have (78). Some examples include "People focus on every word I say," "Everyone in the room will hear me stutter," and "No one will like me if I stutter" (79). Other cognitive and behavioral mechanisms which maintain social anxiety are also modified for the experience of stuttering (11). For example, PWS will often use stutteringspecific safety behaviors such as avoiding and substituting feared words, avoiding anxiety-inducing communicative situations, and rehearsing speech before speaking $(13,80)$. VRET protocols designed for PWS need to consider how social anxiety and the mechanisms that underpin it will differ to ensure maximum relevance of treatment.

\section{Emotional Processing vs. Inhibitory Learning}

Not all studies specified the details of how they conducted exposure, and more specifically what the aim of exposure exercises were. However, there were studies adopting both emotional processing and inhibitory learning methodologies. Two of the studies adopted the emotional processing method (48, 54). This involves targeting anxiety levels throughout exercises with a focus on habituation and gradual exposure to increasingly anxiety-inducing situations in line with an individual's fear hierarchy. Participants in one of these studies rated their anxiety levels before, during and after each exercise, and repeated it another time if anxiety did not decrease (54). Participants in the other study moved onto the next exposure exercise if anxiety had decreased by $50 \%$ across three consecutive attempts or whether ratings before an attempt were sufficiently low (48). The benefit of this approach is that is simple to administer and therefore could have greater potential for delivering VRET at scale.

Two other studies used the inhibitory learning model to inform their treatment design $(47,52)$. Therefore, the aim of these studies was to target participant expectations to develop new non-threatening associations with feared social situations. These kinds of techniques may be well-suited to general social anxiety since it can arise in various situations across varying contexts. Inhibitory learning is proposed as a method to inform new learning of associations across contexts rather than the specific context in which exposure was completed. Thus, varying the context for exposure is also considered important to broaden that learning.

Inhibitory learning may be well-suited to dealing with the negative reactions that are likely to continue post-treatment. At the heart of social anxiety lies the fear of negative evaluation that involves the assumptions of being negatively evaluated, and the overestimation of the consequences this will have. Models of social anxiety suggest that limitations in social performance, which often causes negative evaluation, are a consequence of anxiety $(10,81)$. However, the speech of someone who stutters can be the source of continued negative evaluation throughout life, meaning reconciling these expectations is made particularly difficult. These events may confirm existing expectations, and clash with newly learnt associations. Whilst presenting corrective information is the fundamental method of exposure therapy, the inhibitory learning theory suggests occasionally displaying one's feared expectation can be used as a way of learning how to deal with negative outcomes, and to attenuate subsequent reacquisition of fear (29). The stuttering-specific nature of social anxiety also needs to be considered in this approach. For example, protocols should consider how expectations and safety behaviors will differ amongst PWS and adapt exercises accordingly. The therapist should also emphasize that exposure is targeting expectations and beliefs regarding others' reactions to speech/social performance, rather than expectations of stuttering itself.

\section{Computer-Generated vs. $360^{\circ}$ Video}

Virtual environments can be created in two ways: computergenerated or using $360^{\circ}$ video. Most of the studies used computer-generated environments (50-52, 54-58). This involves environments replicated using software, and computergenerated avatars as people. Some of the older studies used extremely rudimentary environments $(52,55,56)$ involving 2D images overlaid onto low-fidelity backgrounds and less realistic lighting compared to more recent studies (54). This progression in technology has meant greater realism within virtual environments, and scenes can now be manipulated and controlled easily. In the case of VRET for social anxiety, the primary benefit is that the reactions of others in the scene can be controlled. Users are also able to interact with objects within the scene using controllers. Despite this progression, older studies using environments with low levels of realism, have still shown successful reduction of social anxiety $(52,55,56)$.

Recent advances in camera technology have also led to the emerging field of VRET research using $360^{\circ}$ video. Three of the studies in this analysis used this method for creating their virtual environments (46-48). These environments use a spherical video recording of a real environment simulating an anxiety-inducing situation, which is then played back in VR for the purposes of exposure. One of the suggested benefits of this medium is that it is more realistic than computer-generated environments. It is also cheaper and does not require an experienced programmer to create environments.

The concept of presence is key for understanding the comparative value of each of these methods. Presence is defined as the "perceptual illusion" of being physically present and involved within a virtual environment (82). It is also conceptualized as a construct that allows fear to be experienced toward a virtual stimulus (83). Thus, according to the emotional processing theory, presence is important because activation of the fear structure is necessary for successful exposure. Presence is likely to be influential under the inhibitory learning model as well. Greater presence indicates greater involvement with 
the scene which is necessary to attend to anxiety-inducing stimuli for the purpose of extinction learning. Realism does influence presence, however not as much as other factors such as tracking level, stereoscopy, and field of view (84). Recent evidence suggests that computer-generated and $360^{\circ}$ video environments are comparable in terms of presence and ability to induce anxiety (85), suggesting differences in realism did not have an effect. However, this study used passive environments including little social interaction. The main limitation of $360^{\circ}$ video is that users are limited to passive navigation around the environment. This is fine for public-speaking scenarios but can be particularly challenging for developing social environments involving interaction and turn-taking. Controlling the environment based on the user's behavior is possible within computer-generated environments, generating greater agency and intentionality which are theorized to influence presence (86). Findings have also shown that whilst presence does not influence VRET outcome in the treatment of social anxiety, the involvement factor does (87). Both methods have shown sufficient presence levels and have been used to successfully reduce levels of social anxiety in VRET. However, computergenerated environments may be more suited to targeting social interaction as part of social anxiety treatment.

\section{Facial Expressions}

Most studies manipulated audience and avatars' facial expressions to vary task difficulty $(46,50-52,55,56)$. This is a common approach to targeting fear of negative evaluation, particularly within public-speaking scenarios. However, it is unclear how effective these VRET protocols have been at reducing fear of negative evaluation. Some of these studies have shown superiority of VRET over waitlist control $(46,52,56)$, but this is not the case for all studies (50). The absence of facial expressions in another study's VRET protocol were suggested to potentially contribute to a lack of fear of negative evaluation effect, whilst in vivo exposure did successfully reduce it (54). The findings suggest standalone exposure can target fear of negative evaluation, but factors within the virtual presentation disrupted this. The authors suggest that facial expressions may be more influential in one-to-one interactions than in audience settings, like those used in the above study reporting null findings (50), where they may be more difficult to register.

The use of $360^{\circ}$ video may support the reduction of fear of negative evaluation through presenting clearer images of facial expressions. Findings from one study showed that exposure to a virtual audience led to significantly greater reductions in fear of negative evaluation than exposure to an empty room (46). This suggests that the inclusion of fear-relevant stimuli, including facial expressions, benefits fear of negative evaluation reduction, even in a public speaking context. The authors suggest that using $360^{\circ}$ video meant audience faces were easier to register. Thus, the inclusion of facial expressions is likely to be an important factor for targeting fear of negative evaluation, so long as faces can be registered. In computer-generated environments, this may be more challenging for public-speaking contexts due to limitations in computer graphics.
Facial feedback is also likely to be an important factor for PWS. However, exposure to positive feedback may be particularly advantageous given PWS tend to avoid positive feedback from others to a greater extent than fluent speakers (88). Exposure to scenarios including positive feedback are commonplace, especially in protocols adopting the inhibitory learning method as this aims to teach new associations with such situations.

\section{HMD Type}

The type of HMD used in VRET will be highly influential in determining how immersive exposure exercises are. HMDs have also substantially improved across the last few decades to include wider field of view, higher-definition images, and controllers to interact with virtual environments. These are factors known to influence presence (84), making them likely to improve VRET outcomes. One of the earlier studies in this review did not use a HMD at all, displaying the virtual environments to participants on a computer screen (55). Participants moved around the environments using a mouse. However, this is now uncommon given the availability of HMDs which provide a far more immersive experience.

Most of the studies used older research-grade HMDs, such as the eMagin $\mathrm{z} 800$ and nVisor SX, which are not available for consumer use $(52-54,56-58)$. However, this is no longer necessary given the quality and price of consumer HMDs. The Pico Goblin used by one of the more recent studies has a wider field of view and greater screen resolution than all of the above HMDs (48). Traditionally, HMDs have needed computers to provide the computational power required for VR. However, the Pico Goblin is one example of a standalone HMD which does not require this. The commercialization of VR technology has made this equipment more accessible both to researchers and users.

Further development in smartphone capabilities mean they are also powerful enough to run VR programs as a standalone HMD. When paired with a smartphone-based HMD, it displays an image to each eye through the phone's screen to create an immersive experience. The phone's gyroscope controls head movement around the scene. Two studies used smartphone HMDs $(46,47)$. Both used the Samsung Gear VR HMD with Samsung smartphones. One of these studies also used a public speaking app to conduct exposure exercises (47). Findings suggest smartphone VRET can be effective at reducing social anxiety, opening up the prospect of any individual owning a VR device to conduct VRET.

All HMDs have demonstrated suitability for delivering VRET targeting social anxiety. More modern HMDs have the added benefit of higher specs which may contribute to greater presence (e.g., interaction using controllers). Smartphone HMDs are not currently at the same standard but are becoming increasingly sophisticated. The choice of HMD will likely depend on factors such as budget and delivery of treatment (e.g., remote vs. inperson).

\section{Therapist vs. Virtual Therapist}

Therapeutic alliance refers to the extent of collaboration and purposeful action between therapist and patient (89) and is considered key for treatment success. The role of the therapist 
is to provide encouragement, guidance through treatment exercises, and to develop the patient's trust and investment in the treatment process (89). However, inconsistent findings suggest the influence of therapeutic alliance on treatment outcome is more complicated in a social anxiety context (90). Some scholars suggest that as fear of negative evaluation is a core element of social anxiety, the interpersonal relationship a patient has with their therapist might itself induce anxiety which disrupts the link to symptom change (91).

A therapist was used in most studies (47, 50-58). The level of training varied between studies, with some using clinical psychology students $(52,57,58)$, some using trained clinical psychologists $(53,55)$, and some using a combination $(47,50$, 51, 54). Responsibilities included guiding the patient through treatment, manipulating tasks according to individual fears, helping to process emotions and thoughts during exposure exercises, and providing encouragement. One study found that therapeutic alliance was a significant predictor of later symptom change in participants receiving VRET (52). Another study also found no difference in therapeutic alliance levels between the VRET and in vivo exposure conditions (50). These findings therefore suggest therapeutic alliance may play a more influential role in the treatment of social anxiety when using VRET.

One study included no therapist (46). A researcher was present throughout exposure exercises, but they dealt with the practicalities of administering exposure exercises. It is unclear what guidance participants received and whether other traditional therapist responsibilities were covered in other ways. This approach is uncommon, and research suggests the lack of therapist contact can result in lower treatment efficacy and adherence to treatment $(92,93)$.

More recent research has investigated eliminating the therapist and replicating them virtually, aided by the development of standalone and smartphone HMDs. Embodied conversational agents can either appear visually or provide guidance through audio (94). The benefit of such self-guided treatments is that they are likely to be far more scalable than existing VRET protocols (95). This approach may also be particularly suitable for treating social anxiety as it eliminates all interpersonal elements of VRET. One study used virtual therapist-guided VRET, comparing it to waitlist (48). The virtual therapist used voiceover to outline principles of exposure, provide instructions and to coach participants through exercises. However, sessions were conducted in-person and selection of scenes was controlled by a researcher. Findings showed that this mode of delivery can reduce social anxiety significantly compared to a waitlist control. These promising findings add further support to the use of consumer technology in VRET.

Using automated self-guided treatments may be particularly appropriate in the case of stuttering, especially for integrating into speech therapy. This technique would cut the additional training a speech therapist requires, making it an efficient way of delivering psychological treatment alongside speech therapy. Findings from one trial demonstrate that automated CBT can aid the long-term maintenance of speech outcomes when integrated into speech restructuring (22). However, findings regarding social anxiety and fear of negative evaluation were less conclusive.
Another study adopting the same automated version of CBT found it was equally as effective as in vivo CBT at reducing social anxiety levels (23). The benefit of self-guided VRET in this context is that behavioral treatment can be prescribed and completed remotely.

However, self-guided VRET could also work as a standalone treatment that is not delivered alongside speech therapy or prescribed by it. For some, the intensive nature of speech therapy can be a barrier to accessing psychological treatment simultaneously (96). Self-guided VRET might also be particularly relevant for those who do not have a diagnosis of SAD but have subclinical levels of social anxiety. PWS with subclinical levels of social anxiety will not typically receive clinical treatment, and they might not approach anyone regarding their anxiety. However, they may still benefit from psychological treatment, and self-guided VRET which can be delivered remotely and administered by themselves may be appropriate. Nevertheless, PWS also report that a pre-existing therapist relationship facilitates accessing psychological treatment alongside speech therapy (96). Therapeutic alliance has also been shown to be influential for speech therapy outcomes (97). Further research is therefore required to understand whether self-guided VRET is suitable and effective for PWS, and whether therapeutic alliance can be achieved with a virtual therapist.

\section{CBT vs. Standalone Exposure}

Earlier VRET research investigated the efficacy of VRET when included into a wider CBT protocol (50-52, 56-58). All of these studies except one (56) compared the VR version of CBT to CBT using in vivo or imaginal exposure. Findings suggest that VR and in vivo CBT are largely comparable, however some evidence points to non-VR CBT treatments as more effective long-term $(51,57)$. It is difficult though to conclude the efficacy of VRET itself from these findings as its effects cannot be separated from the effects of cognitive treatment elements.

Delivering VRET as a standalone treatment is another way of streamlining treatment to make it less resource-heavy and more accessible. Several studies have eliminated the cognitive elements of treatment in their VRET protocols $(46,47,53,54)$. As such, the number of sessions they included was generally lower, ranging from 1 to 10 sessions. Only one of these studies compared VRET against another treatment format, finding that VRET was comparable to in vivo exposure at reducing social anxiety (54). However, findings also showed VRET did not reduce fear of negative evaluation, whilst in vivo treatment did. More recent findings show standalone VRET can reduce fear of negative evaluation $(46,47)$, suggesting this is not an issue for exposure conducted in VR.

However, no studies have compared standalone VRET to CBT with VRET, making it difficult to decipher the influence of cognitive elements of treatment conducted in a VR context. Previous findings suggest standalone exposure is comparable to CBT when delivered in vivo (26). However, authors from one of the analyzed studies make the suggestion that targeting cognitions may be more important in the context of VR, given users can make use of cognitive avoidance strategies [e.g., "the virtual social world is not real so I do not need to be afraid"; (54)]. 
The current findings outlined above suggest that VRET does have the potential for delivery as an effective standalone treatment, but it is not possible to conclude how its efficacy compares to virtual delivery of CBT.

\section{DISCUSSION}

The aim of this scoping review was to investigate design considerations for VRET protocols targeting social anxiety associated with stuttering, given the lack of research in this area. We reviewed 12 studies using VRET to reduce social anxiety, in order to understand key distinctions in treatment protocols. Based on the findings of the scoping review, we discussed how VRET could be adapted to stuttering, and informed suggestions for future research in this area.

As our findings indicate, there are several notable distinctions in how treatment protocols are designed. The choice of treatment features and protocol design will likely depend on the circumstance. For example, VRET for public speaking anxiety has shown effectiveness in reducing social anxiety levels, whereas a protocol targeting broader social fears will be more appropriate for someone experiencing general social anxiety. Similarly, the emotional processing and inhibitory learning approaches to exposure have both shown success in VRET, and each will be more suitable in particular contexts. Whilst inhibitory learning techniques are principally suited to phasing out safety behaviors and treating anxiety across a variety of contexts, the emotional processing approach is far simpler to administer, making it well-suited for automated VRET. The other notable finding is the adoption of new technology. Recent advances in $360^{\circ}$ video quality have made it possible to create virtual environments with greater ease. Additionally, standalone and smartphone HMDs have progressed the development of automated VRET guided by a virtual therapist. The benefit of these various approaches is that VRET can be adapted for different contexts.

PWS may benefit from some of the protocols examined in the reviewed studies but adapting VRET to stutteringspecific fears will ensure it considers the unique experience of comorbid stuttering and social anxiety. However, a general limitation of VRET is the difficulty of personalizing exercises to individual fears, as exercises must be created in advance. Exposure exercises should be adapted to target key stutteringrelated themes such as telephone speaking and word substitution, but individual fears, safety behaviors and beliefs will vary. Nevertheless, we believe several VRET techniques adopted in previous protocols could be used in VRET designed for PWS. The inhibitory learning method may be appropriate for integrating individual expectations and safety behaviors into exposure exercises. This approach might also be particularly suitable for targeting reacquisition of fear, which is a greater risk for PWS considering the continued reactions from others in relation to speech. Given the challenges of adapting VRET to stuttering and the varied experiences of social anxiety across this group, it is key that PWS are involved in the design process to ensure maximum relevance.
Of particular interest to stuttering-specific VRET is automation and the use of virtual therapists. This may be a more approachable technique for individuals whose primary fears revolve around communication and social evaluationfears which may arise in the presence of a therapist (98). This could also be suitable for PWS who experience subclinical social anxiety. However, development of self-guided VRET is in its early stages and only a handful of studies have adopted virtual therapists for social anxiety (48) and other types of anxiety (99-101). Further research is required to explore the applicability of these techniques. Successfully recreating the therapist role will be integral to remote delivery of VRET, and therapeutic alliance is typically an influential factor in other stuttering treatments. Whilst some evidence supports the therapeutic relationship in VRET $(50,102)$, no study has explored whether similar levels of therapeutic alliance can be achieved with a virtual therapist. As highlighted previously, the relationship between therapeutic alliance and treatment outcome is not straightforward for social anxiety $(52,90,91)$. However, given that virtual therapist-guided VRET eliminates the human social exchange, therapeutic alliance might be bolstered in this format. More research is required to explore whether these techniques are suitable and effective for PWS as well as the influence of different embodiments of the virtual therapist.

Another suggestion is to include VRET in speech therapy. Whilst this approach might not yield benefits to social anxiety, it is expected to aid retention of benefits from speech therapy and increase the uptake of social anxiety treatment amongst PWS. Future research could also target the intertwined issues associated with comorbid stuttering and social anxiety by integrating VRET and Acceptance and Commitment Therapy (ACT). Whilst exposure therapy focuses on eliminating or inhibiting fear and distressing thoughts, ACT targets the consequent struggle to control or eliminate these thoughts. Symptom reduction is therefore not a primary aim of ACT; rather, it encourages the patient to fully experience and embrace anxiety by teaching acceptance of these thoughts and feelings (103). For PWS, anxiety revolves around their stutter and negative self-perceptions of their speech (11), which contributes to greater speech dissatisfaction (12). Treatments which promote self-efficacy are also important for long-term improvement (104). When combined with VRET, ACT may aid treatment engagement and reduce safety behaviors (105), whilst targeting the broader experience of negative thoughts surrounding stuttering. Research supports the use of ACT to aid maintenance of benefits from speech therapy and reduce the adverse impact of stuttering on participants' lives (106). Findings from two other studies support the use of ACT in the wider population to reduce social anxiety, demonstrating equal effectiveness with CBT $(107,108)$. Research has already experimented with combining VRET and ACT for treating social anxiety amongst fluent speakers, suggesting this might be a suitable technique for reducing social anxiety (109). More research is required to understand the contribution of ACT to treatment outcome when combined with VRET, and how such a combination of treatments compares to existing social anxiety treatments. Lastly, research should also seek to understand whether this can be effective in PWS. 
More research is also required to understand the processes that make VRET effective. Presence is another concept that is theorized to have a significant influence on treatment outcome and experiencing anxiety virtually. Whilst presence appears to be a strong predictor of anxiety for most anxiety disorders, it is not clear whether this relationship exists at all for $\operatorname{SAD}(34,110,111)$. It is suggested that the constructs that make up presence may be less relevant to experiencing fear of negative evaluation, which is triggered by social cues. As such, presence may also have less of an impact on treatment effectiveness and symptom change (87). Attention has also turned to social presence, which is likely to play a more significant role within social virtual environments. Social presence is conceptually distinct from physical presence (112) and describes the sensation of being in the presence of another being, which requires a level of cognitive and emotional engagement. No study to date has investigated the relationship between social presence and VRET outcomes, but SAD patients have shown heightened co-presence and mutual attention in response to virtual environments (33), suggesting it might be influential. These links require further investigation to inform the treatment features and modalities necessary for treating social anxiety.

\section{Review Limitations}

There are several limitations to the current scoping review. First, as we did not conduct a meta-analysis or any other quantitative analysis, we cannot directly compare the effectiveness of different treatments and the techniques they use. A scoping review approach was chosen based on the relatively small literature on VRET protocols for social anxiety and PWS, and to provide a qualitative overview of these protocols. Second, there were inconsistencies and missing details in the reporting of VRET protocols, study methods and findings. As a result, there may have been details that were missed in the analyses. This is a common limitation, however, and points to the importance of creating standardized reporting methods for interventions contributing to reproducibility as well as replicability of findings in this area. Third, several studies investigating VRET for social anxiety were not included in the review as they were outside of the scoping criteria. Some of these expand on innovative techniques such as cloud-based VRET (113), or broaden our understanding of VRET effectiveness by using functional magnetic resonance imaging (114), yet the cumulative effects of such protocols are still unknown due to their recency. Finally, this review focused on adults due to differences in developmental trajectories of social anxiety as well as limited availability of protocols for children and adolescents. Nonetheless, more research is required on the usefulness and efficacy of VRET for non-adult populations.

\section{Review Strengths}

This scoping review represents the first comprehensive overview of the various techniques and approaches used in VRET protocols targeting social anxiety. To our knowledge, this is also the first scoping review to focus on exposure protocols designed for PWS. Our findings provide a deeper understanding of the comparative value of different VRET approaches, and how these can be used to suit different circumstances. Furthermore, all reviewed studies compared VRET against another treatment or control condition, allowing greater confidence in the conclusions drawn about the efficacy of different protocols.

\section{CONCLUSION}

The past decade has seen an increased interest in using virtual reality for mental healthcare. This has coincided with the rising availability of commercial VR headsets, paired with overwhelmed mental healthcare systems across the globe. Yet there is much work to be done regarding systematic understanding of VR intervention protocols, specifically for certain groups such as PWS. Due to a lack of research on the suitability of VRET for PWS, it is difficult to draw conclusions about the effectiveness of these techniques. The current review suggests several techniques that may be appropriate in the case of stuttering that need further testing, including automated VRET using a virtual therapist, inhibitory learning techniques, and integration with other treatments. Regardless of the approach taken, it is key that VRET is adapted to the nature of social anxiety associated with stuttering. Finally, our review has also highlighted the need for creating, sharing, and testing VR intervention protocols in a systematic manner, to increase reproducibility as well as replicability of findings.

\section{AUTHOR CONTRIBUTIONS}

IC: conceptualization, literature search and analysis, and writing original draft. IC and NZ: writing reviews and editing. NZ: supervision. Both authors contributed to the article and approved the submitted version.

\section{FUNDING}

The research was funded by a training grant from UK Research and Innovation and Imperial College London (no. EP/R513052/1).

\section{ACKNOWLEDGMENTS}

A preprint of this review paper is available on PsyArXiv (doi: 10.31234/osf.io/bywe5).

\section{SUPPLEMENTARY MATERIAL}

The Supplementary Material for this article can be found online at: https://www.frontiersin.org/articles/10.3389/fdgth. 2022.842460/full\#supplementary-material 


\section{REFERENCES}

1. American Psychiatric Association. Diagnostic and Statistical Manual of Mental Disorders, 5th Edn. Washington, DC: American Psychiatric Association (2013). doi: 10.1176/appi.books.9780890425596

2. Craig A, Hancock K, Tran Y, Craig M, Peters K. Epidemiology of stuttering in the community across the entire life span. J Speech Lang Hear Res. (2002) 45:1097-105. doi: 10.1044/1092-4388(2002/088)

3. Howell P. Language Processing in Fluency Disorders. In: Guendouzi J, Loncke F, Williams MJ, editors. The Handbook on Psycholinguistics and Cognitive Processes: Perspectives on Communication Disorders. New York, NY: Psychology Press (2010). p. 437-64.

4. Bloodstein O, Bernstein Ratner N. A Handbook on Stuttering, 6th Edn. Clifton Park, NY: Thomson/Delmar Learning (2008).

5. Yairi E, Ambrose N. Epidemiology of stuttering: 21st century advances. $J$ Fluency Disord. (2013) 38:66-87. doi: 10.1016/j.jfludis.2012.11.002

6. Iverach L, Rapee RM. Social anxiety disorder and stuttering: current status and future directions. J Fluency Disord. (2014) 40:69-82. doi: 10.1016/j.jfludis.2013.08.003

7. Craig A, Tran Y. Trait and social anxiety in adults with chronic stuttering: Conclusions following meta-analysis. J Fluency Disord. (2014) 40:3543. doi: 10.1016/j.jfludis.2014.01.001

8. Dalrymple KL, Zimmerman M. When does benign shyness become social anxiety, a treatable disorder? Curr Psychiatry. (2013) 12:21-3, 35-8. Available online at: https://cdn.mdedge.com/files/s3fs-public/Document/September2017/021_1113CP_Dalrymple_FINAL.pdf

9. Blumgart E, Tran Y, Craig A. Social anxiety disorder in adults who stutter. Depress Anxiety. (2010) 27:687-92. doi: 10.1002/da.20657

10. Rapee RM, Heimberg RG. A cognitive-behavioral model of anxiety in social phobia. Behav Res Ther. (1997) 35:74156. doi: 10.1016/S0005-7967(97)00022-3

11. Iverach L, Rapee RM, Wong QJJ, Lowe R. Maintenance of social anxiety in stuttering: a cognitive-behavioral model. Am J Speech Lang Pathol. (2017) 26:540-56. doi: 10.1044/2016_AJSLP-16-0033

12. Iverach L, Jones M, Lowe R, O'Brian S, Menzies RG, Packman A, et al. Comparison of adults who stutter with and without social anxiety disorder. $J$ Fluency Disord. (2018) 56:55-68. doi: 10.1016/j.jfludis.2018.03.001

13. Lowe R, Helgadottir F, Menzies R, Heard R, O'Brian S, Packman A, et al. Safety behaviors and stuttering. J Speech Lang Hear Res. (2017) 60:124653. doi: 10.1044/2016_JSLHR-S-16-0055

14. Menzies RG, O'Brian S, Onslow M, Packman A, St Clare T, Block S. An experimental clinical trial of a cognitive-behavior therapy package for chronic stuttering. J Speech Lang Hear Res. (2008) 51:1451-64. doi: 10.1044/1092-4388(2008/07-0070)

15. Tolin DF. Is cognitive-behavioral therapy more effective than other therapies?: a meta-analytic review. Clin Psychol Rev. (2010) 30:71020. doi: 10.1016/j.cpr.2010.05.003

16. Maxwell DL. Cognitive and behavioral self-control strategies: applications for the clinical management of adult stutterers. J Fluency Disord. (1982) 7:403-32. doi: 10.1016/0094-730X(82)90018-3

17. Blood GW. Power2: Relapse management with adolescents who stutter. Lang Speech Hear Serv Schools. (1995) 26:16979. doi: 10.1044/0161-1461.2602.169

18. Stein MB, Baird A, Walker JR. Social phobia in adults with stuttering. Am J Psychiatry. (1996) 153:278-80. doi: 10.1176/ajp.153.2.278

19. Gunn A, Menzies RG, Onslow M, O’Brian S, Packman A, Lowe R, et al. Phase I trial of a standalone internet social anxiety treatment for adolescents who stutter: iBroadway. Int J Lang Commun Disord. (2019) 54:927-39. doi: 10.1111/1460-6984.12496

20. Helgadóttir FD, Menzies RG, Onslow M, Packman A, O’Brian S. Online CBT II: a phase I trial of a standalone, online CBT treatment program for social anxiety in stuttering. Behav Change. (2009) 26:254-70.

21. Helgadóttir FD, Menzies RG, Onslow M, Packman A, O’Brian S. A standalone Internet cognitive behavior therapy treatment for social anxiety in adults who stutter: CBTpsych. J Fluency Disord. (2014) 41:4754. doi: 10.1016/j.jfludis.2014.04.001

22. Menzies RG, O’Brian S, Packman A, Jones M, Helgadóttir FD, Onslow M. Supplementing stuttering treatment with online cognitive behavior therapy: an experimental trial. J Commun Disord. (2019) 80:81-91. doi: 10.1016/j.jcomdis.2019.04.003

23. Menzies RG, Packman A, Onslow M, O’Brian S, Jones M, Helgadóttir FD. In-clinic and standalone internet cognitive behavior therapy treatment for social anxiety in stuttering: a randomized trial of iGlebe. J Speech Lang Hear Res. (2019) 62:1614-24. doi: 10.1044/2019_JSLHR-S-18-0340

24. Menzies RG, O’Brian S, Lowe R, Packman A, Onslow M. International phase II clinical trial of CBTPsych: a standalone internet social anxiety treatment for adults who stutter. J Fluency Disord. (2016) 48:3543. doi: 10.1016/j.jfludis.2016.06.002

25. Rodebaugh TL, Holaway RM, Heimberg RG. The treatment of social anxiety disorder. Clin Psychol Rev. (2004) 24:883-908. doi: 10.1016/j.cpr.2004.07.007

26. Powers MB, Sigmarsson SR, Emmelkamp PMG. A meta-analytic review of psychological treatments for social anxiety disorder. Int J Cogn Ther. (2008) 1:94-113. doi: 10.1521/ijct.2008.1.2.94

27. Foa EB, Kozak MJ. Emotional processing of fear - exposure to corrective information. Psychol Bull. (1986) 99:20-35. doi: 10.1037/0033-2909. 99.1.20

28. Craske MG, Kircanski K, Zelikowsky M, Mystkowski J, Chowdhury N, Baker A. Optimizing inhibitory learning during exposure therapy. Behav Res Ther. (2008) 46:5-27. doi: 10.1016/j.brat.2007.10.003

29. Craske MG, Treanor M, Conway CC, Zbozinek T, Vervliet B. Maximizing exposure therapy: an inhibitory learning approach. Behav Res Ther. (2014) 58:10-23. doi: 10.1016/j.brat.2014.04.006

30. Hartanto D, Kampmann IL, Morina N, Emmelkamp PGM, Neerincx MA, Brinkman WP. Controlling social stress in virtual reality environments. PLoS ONE. (2014) 9:e0223988. doi: 10.1371/journal.pone.0092804

31. Owens ME, Beidel DC. Can virtual reality effectively elicit distress associated with social anxiety disorder? J Psychopathol Behav Assess. (2015) 37:296305. doi: 10.1007/s10862-014-9454-x

32. Barreda-Angeles M, Aleix-Guillaume S, Pereda-Banos A. Users' psychophysiological, vocal, and self-reported responses to the apparent attitude of a virtual audience in stereoscopic 360 degrees-video. Virtual Real. (2020) 24:289-302. doi: 10.1007/s10055-019-00400-1

33. Felnhofer A, Hlavacs H, Beutl L, Kryspin-Exner I, Kothgassner OD. Physical presence, social presence, and anxiety in participants with social anxiety disorder during virtual cue exposure. Cyberpsychol Behav Soc Netw. (2019) 22:46-50. doi: 10.1089/cyber.2018.0221

34. Felnhofer A, Kothgassner OD, Hetterle T, Beutl L, Hlavacs H, Kryspin-Exner I. Afraid to be there? Evaluating the relation between presence, self-reported anxiety, and heart rate in a virtual public speaking task. Cyberpsychol Behav Soc Netw. (2014) 17:310-6. doi: 10.1089/cyber.2013.0472

35. Lange B, Pauli P. Social anxiety changes the way we move - A social approach-avoidance task in a virtual reality CAVE system. PLoS ONE. (2019) 14:e0226805. doi: 10.1371/journal.pone.0226805

36. Rubin M, Minns S, Muller K, Tong MH, Hayhoe MM, Telch MJ. Avoidance of social threat: evidence from eye movements during a public speaking challenge using $360^{\circ}$ - video. Behav Res Ther. (2020) 134:103706. doi: 10.1016/j.brat.2020.103706

37. Scheurich JA, Beidel DC, Vanryckeghem M. Exposure therapy for social anxiety disorder in people who stutter: an exploratory multiple baseline design. J Fluency Disord. (2019) 59:21-32. doi: 10.1016/j.jfludis.2018.12.001

38. Walkom G, editor. Virtual reality exposure therapy: to benefit those who stutter and treat social anxiety. In: 2016 International Conference on Interactive Technologies and Games (ITAG). Nottingham (2016). doi: 10.1109/iTAG.2016.13

39. Kampmann IL, Emmelkamp PMG, Morina N. Meta-analysis of technologyassisted interventions for social anxiety disorder. J Anxiety Disord. (2016) 42:71-84. doi: 10.1016/j.janxdis.2016.06.007

40. Chesham RK, Malouff JM, Schutte NS. Meta-analysis of the efficacy of virtual reality exposure therapy for social anxiety. Behav Change. (2018) 35:152-66. doi: 10.1017/bec.2018.15

41. Horigome T, Kurokawa S, Sawada K, Kudo S, Shiga K, Mimura $\mathrm{M}$, et al. Virtual reality exposure therapy for social anxiety disorder: a systematic review and meta-analysis. Psychol Med. (2020) 50:248797. doi: $10.1017 /$ S0033291720003785

42. Carl E, Stein AT, Levihn-Coon A, Pogue JR, Rothbaum B, Emmelkamp $\mathrm{P}$, et al. Virtual reality exposure therapy for anxiety and related disorders: 
a meta-analysis of randomized controlled trials. J Anxiety Disord. (2019) 61:27-36. doi: 10.1016/j.janxdis.2018.08.003

43. Fodor LA, Coteț CD, Cuijpers P, Szamoskozi Ş, David D, Cristea IA. The effectiveness of virtual reality based interventions for symptoms of anxiety and depression: a meta-analysis. Sci Rep. (2018) 8:10323. doi: 10.1038/s41598-018-28113-6

44. Opris D, Pintea S, Garcia-Palacios A, Botella C, Szamoskozi S, David D. Virtual reality exposure therapy in anxiety disorders: a quantitative metaanalysis. Depress Anxiety. (2012) 29:85-93. doi: 10.1002/da.20910

45. Wechsler TF, Mühlberger A, Kümpers F. Inferiority or even superiority of virtual reality exposure therapy in phobias? - A systematic review and quantitative meta-analysis on randomized controlled trials specifically comparing the efficacy of virtual reality exposure to gold standard in vivo exposure in Agoraphobia, Specific Phobia and Social Phobia. Front Psychol. (2019) 10:1758. doi: 10.3389/fpsyg.2019.01758

46. Reeves R, Elliott A, Curran D, Dyer K, Hanna D. $360^{\circ}$ Video virtual reality exposure therapy for public speaking anxiety: A randomized controlled trial. J Anxiety Disord. (2021) 83:102451. doi: 10.1016/j.janxdis.2021.102451

47. Lindner P, Miloff A, Fagernas S, Andersen J, Sigeman M, Andersson G, et al. Therapist-led and self-led one-session virtual reality exposure therapy for public speaking anxiety with consumer hardware and software: a randomized controlled trial. J Anxiety Disord. (2019) 61:4554. doi: 10.1016/j.janxdis.2018.07.003

48. Zainal NH, Chan WW, Saxena AP, Barr Taylor C, Newman MG. Pilot randomized trial of self-guided virtual reality exposure therapy for social anxiety disorder. Behav Res Ther. (2021) 147:103984. doi: 10.1016/j.brat.2021.103984

49. Tricco AC, Lillie E, Zarin W, O’Brien KK, Colquhoun H, Levac D, et al. PRISMA Extension for Scoping Reviews (PRISMA-ScR): checklist and explanation. Ann Intern Med. (2018) 169:467-73. doi: 10.7326/M18-0850

50. Anderson PL, Price M, Edwards SM, Obasaju MA, Schmertz SK, Zimand E, et al. Virtual reality exposure therapy for social anxiety disorder: a randomized controlled trial. J Consult Clin Psychol. (2013) 81:75160. doi: $10.1037 / \mathrm{a} 0033559$

51. Anderson PL, Edwards SM, Goodnight JR. Virtual reality and exposure group therapy for social anxiety disorder: results from a 4-6 year follow-up. Cogn Ther Res. (2017) 41:230-6. doi: 10.1007/s10608-016-9820-y

52. Bouchard S, Dumoulin S, Robillard G, Guitard T, Klinger E, Forget H, et al. Virtual reality compared with in vivo exposure in the treatment of social anxiety disorder: a three-arm randomised controlled trial. Br J Psychiatry. (2017) 210:276-83. doi: 10.1192/bjp.bp.116.184234

53. Harris SR, Kemmerling RL, North MM. Brief virtual reality therapy for public speaking anxiety. Cyberpsychol Behav. (2002) 5:543-50. doi: 10.1089/109493102321018187

54. Kampmann IL, Emmelkamp PMG, Hartanto D, Brinkman WP, Zijlstra BJH, Morina N. Exposure to virtual social interactions in the treatment of social anxiety disorder: a randomized controlled trial. Behav Res Ther. (2016) 77:147-56. doi: 10.1016/j.brat.2015.12.016

55. Klinger E, Bouchard S, Legeron P, Roy S, Lauer F, Chemin I, et al. Virtual reality therapy versus cognitive behavior therapy for social phobia: a preliminary controlled study. Cyberpsychol Behav. (2005) 8:7688. doi: $10.1089 / \mathrm{cpb} .2005 .8 .76$

56. Robillard G, Bouchard S, Dumoulin S, Guitard T, Klinger É. Using virtual humans to alleviate social anxiety: preliminary report from a comparative outcome study. Stud Health Technol Inform. (2010) 154:57-60. doi: 10.3233/978-1-60750-561-7-57

57. Safir MP, Wallach HS, Bar-Zvi M. Virtual reality cognitive-behavior therapy for public speaking anxiety: one-year follow-up. Behav Modif. (2012) 36:23546. doi: 10.1177/0145445511429999

58. Wallach HS, Safir MP, Bar-Zvi M. Virtual reality cognitive behavior therapy for public speaking anxiety: a randomized clinical trial. Behav Modif. (2009) 33:314-38. doi: 10.1177/0145445509331926

59. Leary MR. A brief version of the fear of negative evaluation scale. Pers Soc Psychol Bull. (1983) 9:371-5. doi: 10.1177/0146167283093007

60. Paul GL. Insight vs. Desensitization in Psychotherapy: An Experiment in Anxiety Reduction. Stanford, CA: Stanford University Press (1966). doi: 10.1097/00000441-196610000-00031
61. Baker SL, Heinrichs N, Kim H-J, Hofmann SG. The Liebowitz social anxiety scale as a self-report instrument: a preliminary psychometric analysis. Behav Res Ther. (2002) 40:701-15. doi: 10.1016/S0005-7967(01)00060-2

62. Safren SA, Turk CL, Heimberg RG. Factor structure of the social interaction anxiety scale and the social phobia scale. Behav Res Ther. (1998) 36:44353. doi: 10.1016/S0005-7967(98)00032-1

63. Watson D, Friend R. Measurement of social-evaluative anxiety. J Consult Clin Psychol. (1969) 33:448-57. doi: 10.1037/h0027806

64. Fydrich T, Chambless DL, Perry KJ, Buergener F, Beazley MB. Behavioral assessment of social performance: a rating system for social phobia. Behav Res Ther. (1998) 36:995-1010. doi: 10.1016/S0005-7967(98)00069-2

65. Liebowitz MR. Social phobia. Modern Probl Pharmacopsychiatry. (1987) 22:141-73. doi: 10.1159/000414022

66. Rathus SA. A 30-item schedule for assessing assertive behavior. Behav Therpy. (1973) 4:398-406. doi: 10.1016/S0005-7894(73)80120-0

67. Bartholomay EM, Houlihan DD. Public speaking anxiety scale: preliminary psychometric data and scale validation. Pers Individ Diff. (2016) 94:2115. doi: 10.1016/j.paid.2016.01.026

68. Telch MJ, Lucas RA, Smits JAJ, Powers MB, Heimberg R, Hart T. Appraisal of social concerns: a cognitive assessment instrument for social phobia. Depress Anxiety. (2004) 19:217-24. doi: 10.1002/da.20004

69. Hofmann SG, DiBartolo PM. An instrument to assess selfstatements during public speaking: scale development and preliminary psychometric properties. Behav Ther. (2000) 31:499-515. doi: 10.1016/S0005-7894(00)80027-1

70. Newman MG, Kachin KE, Zuellig AR, Constantino MJ, Cashman-McGrath L. The social phobia diagnostic questionnaire: preliminary validation of a new self-report diagnostic measure of social phobia. Psychol Med. (2003) 33:623-35. doi: 10.1017/S0033291703007669

71. McCarthy J, Goffin R. Measuring job interview anxiety: beyond weak knees and sweaty palms. Pers Psychol. (2004) 57:607-37. doi: 10.1111/j.1744-6570.2004.00002.x

72. Iverach L, Jones $\mathrm{M}$, O'Brian S, Block S, Lincoln M, Harrison E, et al. The relationship between mental health disorders and treatment outcomes among adults who stutter. J Fluency Disord. (2009) 34:2943. doi: 10.1016/j.jfludis.2009.02.002

73. Plexico L, Manning WH, DiLollo A. A phenomenological understanding of successful stuttering management. J Fluency Disord. (2005) 30:122. doi: 10.1016/j.jfludis.2004.12.001

74. Ruscio AM, Brown TA, Chiu WT, Sareen J, Stein MB, Kessler RC. Social fears and social phobia in the USA: results from the National Comorbidity Survey Replication. Psychol Med. (2008) 38:15-28. doi: 10.1017/S0033291707001699

75. Haitani T, Sakai N, Mori K, Houjou T. Situational factors influencing social anxiety in treatment-seeking adults who stutter: an exploratory factor analysis. PsyArXiv [Preprint] (2021). Available online at: https://psyarxiv. com/h3957/ (accessed December 14, 2021).

76. Haitani T, Sakai N, Mori K, Houjou T. Heterogeneity of social anxiety in treatment-seeking adults who stutter: A latent profile analysis. PsyArXiv [Preprint] (2021). Available online at: https://psyarxiv.com/8sc7d/ (accessed December 14, 2021).

77. Iverach L, O'Brian S, Jones $\mathrm{M}$, Block S, Lincoln $\mathrm{M}$, Harrison E, et al. Prevalence of anxiety disorders among adults seeking speech therapy for stuttering. J Anxiety Disord. (2009) 23:92834. doi: 10.1016/j.janxdis.2009.06.003

78. Iverach L, Menzies R, Jones M, O’Brian S, Packman A, Onslow M. Further development and validation of the Unhelpful Thoughts and Beliefs About Stuttering (UTBAS) scales: relationship to anxiety and social phobia among adults who stutter. Int J Lang Commun Disord. (2010) 46:28699. doi: 10.3109/13682822.2010.495369

79. St Clare T, Menzies RG, Onslow M, Packman A, Thompson R, Block S. Unhelpful thoughts and beliefs linked to social anxiety in stuttering: development of a measure. Int J Lang Commun Disord. (2009) 44:33851. doi: $10.1080 / 13682820802067529$

80. Vanryckeghem M, Brutten GJ, Uddin N, Borsel JV. A comparative investigation of the speech-associated coping responses reported by adults who do and do not stutter. J Fluency Disord. (2004) 29:23750. doi: $10.1016 /$ j.jfludis.2004.07.001 
81. Clark DM, Wells A. A cognitive model of social phobia. Soc Phobia. (1995) 41:63-9.

82. Lombard M, Ditton T. At the heart of it all: the concept of presence. J Comput Mediated Commun. (1997) 3:JCMC321. doi: 10.1111/j.1083-6101.1997.tb00072.x

83. Price $M$, Anderson $P$. The role of presence in virtual reality exposure therapy. J Anxiety Disord. (2007) 21:742-51. doi: 10.1016/j.janxdis.2006.11.002

84. Cummings JJ, Bailenson JN. How immersive is enough? A meta-analysis of the effect of immersive technology on user presence. Media Psychol. (2016) 19:272-309. doi: 10.1080/15213269.2015.1015740

85. Brivio E, Serino S, Negro Cousa E, Zini A, Riva G, De Leo G. Virtual reality and $360^{\circ}$ panorama technology: a media comparison to study changes in sense of presence, anxiety, and positive emotions. Virtual Real. (2020) 25:303-11. doi: 10.1007/s10055-020-00453-7

86. Triberti S, Riva G. Being present in action: a theoretical model about the "interlocking" between intentions and environmental affordances. Front Psychol. (2016) 6:2052. doi: 10.3389/fpsyg.2015.02052

87. Price M, Mehta N, Tone EB, Anderson PL. Does engagement with exposure yield better outcomes? Components of presence as a predictor of treatment response for virtual reality exposure therapy for social phobia. J Anxiety Disord. (2011) 25:763-70. doi: 10.1016/j.janxdis.2011.03.004

88. Lowe R, Guastella AJ, Chen NTM, Menzies RG, Packman A, O’Brian S, et al. Avoidance of eye gaze by adults who stutter. J Fluency Disord. (2012) 37:263-74. doi: 10.1016/j.jfludis.2012.04.004

89. Horvath AO, Luborsky L. The role of the therapeutic alliance in psychotherapy. J Consult Clin Psychol. (1993) 61:56173. doi: 10.1037/0022-006X.61.4.561

90. Jazaieri H, Goldin PR, Gross JJ. The role of working alliance in CBT and MBSR for social anxiety disorder. Mindfulness. (2018) 9:13819. doi: 10.1007/s12671-017-0877-9

91. Draheim AA, Anderson PL. Working alliance does not mediate the relation between outcome expectancy and symptom improvement following cognitive behavioural therapy for social anxiety disorder. Cogn Behav Ther. (2019) 12:e41. doi: 10.1017/S1754470X19000266

92. Andersson G, Titov N, Dear BF, Rozental A, Carlbring P. Internetdelivered psychological treatments: from innovation to implementation. World Psychiatry. (2019) 18:20-8. doi: 10.1002/wps.20610

93. Christensen H, Griffiths KM, Farrer L. Adherence in internet interventions for anxiety and depression: systematic review. J Med Internet Res. (2009) 11:e13. doi: 10.2196/jmir.1194

94. Provoost S, Lau HM, Ruwaard J, Riper H. Embodied conversational agents in clinical psychology: a scoping review. J Med Internet Res. (2017) 19:e151. doi: 10.2196/jmir.6553

95. Fairburn CG, Patel V. The impact of digital technology on psychological treatments and their dissemination. Behav Res Ther. (2017) 88:1925. doi: 10.1016/j.brat.2016.08.012

96. Lindsay A, Langevin M. Psychological counseling as an adjunct to stuttering treatment: clients' experiences and perceptions. J Fluency Disord. (2017) 52:1-12. doi: 10.1016/j.jfludis.2017.01.003

97. Sønsterud H, Kirmess M, Howells K, Ward D, Feragen KB, Halvorsen MS. The working alliance in stuttering treatment: a neglected variable? Int J Lang Commun Disord. (2019) 54:606-19. doi: 10.1111/1460-6984.12465

98. Hayes SA, Hope DA, Van Dyke MM, Heimberg RG. Working alliance for clients with social anxiety disorder: relationship with session helpfulness and within-session habituation. Cogn Behav Ther. (2007) 36:34-42. doi: 10.1080/16506070600947624

99. Miloff A, Lindner P, Dafgård P, Deak S, Garke M, Hamilton W, et al. Automated virtual reality exposure therapy for spider phobia vs. in-vivo onesession treatment: a randomized non-inferiority trial. Behav Res Ther. (2019) 118:130-40. doi: 10.1016/j.brat.2019.04.004

100. Freeman D, Haselton P, Freeman J, Spanlang B, Kishore S, Albery E, et al. Automated psychological therapy using immersive virtual reality for treatment of fear of heights: a single-blind, parallelgroup, randomised controlled trial. Lancet Psychiatry. (2018) 5:625-32. doi: 10.1016/S2215-0366(18)30226-8

101. Donker T, Cornelisz I, van Klaveren C, van Straten A, Carlbring P, Cuijpers P, et al. Effectiveness of self-guided app-based virtual reality cognitive behavior therapy for acrophobia: a randomized clinical trial. JAMA Psychiatry. (2019) 76:682-90. doi: 10.1001/jamapsychiatry.2019.0219

102. Ngai I, Tully EC, Anderson PL. The course of the working alliance during virtual reality and exposure group therapy for social anxiety disorder. Behav Cogn Psychother. (2015) 43:167-81. doi: 10.1017/S13524658130 0088X

103. Hayes SC, Strosahl KD, Wilson KG. Acceptance and Commitment Therapy: An Experiential Approach to Behavior Change. New York, NY: Guilford Press (1999).

104. Craig A. Relapse following treatment for stuttering: a critical review and correlative data. J Fluency Disord. (1998) 23:130. doi: 10.1016/S0094-730X(97)00027-2

105. Eifert GH, Heffner M. The effects of acceptance versus control contexts on avoidance of panic-related symptoms. J Behav Ther Exp Psychiatry. (2003) 34:293-312. doi: 10.1016/j.jbtep.2003.11.001

106. Beilby JM, Byrnes ML, Yaruss JS. Acceptance and commitment therapy for adults who stutter: psychosocial adjustment and speech fluency. J Fluency Disord. (2012) 37:289-99. doi: 10.1016/j.jfludis.2012. 05.003

107. Craske MG, Niles AN, Burklund LJ, Wolitzky-Taylor KB, Plumb-Vilardaga JC, Arch JJ, et al. Randomized controlled trial of cognitive behavioral therapy and acceptance and commitment therapy for social phobia: outcomes and moderators. J Consult Clin Psychol. (2014) 82:103448. doi: $10.1037 / \mathrm{a} 0037212$

108. Kocovski NL, Fleming JE, Hawley LL, Huta V, Antony MM. Mindfulness and acceptance-based group therapy versus traditional cognitive behavioral group therapy for social anxiety disorder: a randomized controlled trial. Behav Res Ther. (2013) 51:889-98. doi: 10.1016/j.brat.2013. 10.007

109. Yuen EK, Goetter EM, Stasio MJ, Ash P, Mansour B, McNally E, et al. A pilot of acceptance and commitment therapy for public speaking anxiety delivered with group videoconferencing and virtual reality exposure. J Contextual Behav Sci. (2019) 12:47-54. doi: 10.1016/j.jcbs.2019. 01.006

110. Ling Y, Nefs HT, Morina N, Heynderickx I, Brinkman WP. A metaanalysis on the relationship between self-reported presence and anxiety in virtual reality exposure therapy for anxiety disorders. PLoS ONE. (2014) 9:e96144. doi: 10.1371/journal.pone.0096144

111. Morina N, Brinkman WP, Hartanto D, Emmelkamp PMG. Sense of presence and anxiety during virtual social interactions between a human and virtual humans. PeerJ. (2014) 2:e337. doi: 10.7717/peerj.337

112. IJsselsteijn WA. Presence in Depth. Eindhoven: Eindhoven University of Technology (2004).

113. Šalkevičius J, Miškinyte A, Navickas L. Cloud based virtual reality exposure therapy service for public speaking anxiety. Information. (2019) 10:62. doi: 10.3390/info10020062

114. Hur J-W, Shin H, Jung D, Lee H-J, Lee S, Kim GJ, et al. Virtual reality-based psychotherapy in social anxiety disorder: fMRI study using a self-referential task. JMIR Ment Health. (2021) 8:e25731. doi: 10.2196/25731

Conflict of Interest: The authors declare that the research was conducted in the absence of any commercial or financial relationships that could be construed as a potential conflict of interest.

Publisher's Note: All claims expressed in this article are solely those of the authors and do not necessarily represent those of their affiliated organizations, or those of the publisher, the editors and the reviewers. Any product that may be evaluated in this article, or claim that may be made by its manufacturer, is not guaranteed or endorsed by the publisher.

Copyright (c) 2022 Chard and van Zalk. This is an open-access article distributed under the terms of the Creative Commons Attribution License (CC BY). The use, distribution or reproduction in other forums is permitted, provided the original author(s) and the copyright owner(s) are credited and that the original publication in this journal is cited, in accordance with accepted academic practice. No use, distribution or reproduction is permitted which does not comply with these terms. 